\title{
Correlação entre a dor lombar e as alterações posturais em gestantes
}

\author{
Correlation between low back pain and postural alterations during pregnancy
}

Paulo José Oliveira Cortez ${ }^{12}$, Ticiane Alves Siniscalchi Franco', Tássia Magalhães Sene', Tatiana Dias de Carvalho ${ }^{3}$, José Elias Tomazini

\begin{abstract}
Resumo
Introdução: A dor lombar é uma queixa comum na população em geral e é sintoma frequentemente relatado durante a gestação. Objetivo: Verificar a correlação entre a dor lombar e as alterações posturais em gestantes. Método: A amostra constituiu-se de 54 voluntárias, com faixa etária variando entre 20 e 30 anos, sendo 27 gestantes. As gestantes foram divididas em três grupos de acordo com o período gestacional: sete no primeiro trimestre (GI), 11 no segundo trimestre (GII), nove no terceiro trimestre (GIII); para ser analisada a incidência de dor ou desconforto relacionada a alterações posturais apresentadas no período gestacional. 0 grupo controle $(\mathrm{GC})$ foi constituído por 27 voluntárias não grávidas. A análise das fotos foi realizada com o software de Análise Postural (SAPO), e a análise estatística foi feita através dos testes ANOVA e Regressão Linear Simples com nível de significância de 5\%. Resultados: Em relação à idade gestacional, a prevalência de dor lombar foi maior nas gestantes com até 13 semanas. Referente à intensidade da dor na região lombar, o Gll foi o grupo que apresentou a maior média, com dor moderada, seguido dos grupos GIIl, GC e Gl, com uma dor leve. Com relação às modificações da curvatura da coluna lombar, não foi possível estabelecer correlação estatisticamente significativa das alterações posturais apresentadas com a presença da dor lombar durante a gestação por meio da análise postural de todas as mulheres. Conclusão: Não houve correlação entre a sintomatologia de dor lombar e as alterações posturais no período gestacional.
\end{abstract}

Palavras-chave: manutenção da gravidez; dor lombar; postura.

\begin{abstract}
Introduction: Low back pain is a common complaint in the population as a whole and is a symptom frequently reported during pregnancy. Objective: To verify the correlation between low back pain and postural changes during pregnancy. Method: The sample consisted of 54 female volunteers, ages from 20 to 30 years, 27 pregnant. Women were sub-divided into three groups according to pregnancy age: seven in first quarter (GI), 11 in the second quarter (GII), nine in third trimester (GIII); to analyze the incidence of pain or discomfort related to abnormal postural alterations during pregnancy. The control group (GC) consisted of 27 non-pregnant volunteers. The image analysis was performed with the Postural Analysis Software (SAPO) and statistical analysis through ANOVA tests and regression analysis with significance level at $5 \%$. Results: Regarding the gestational age, the prevalence of lumbar pain was higher among those with up to 13 weeks. Referring to the intensity of pain in the lumbar region, the Gll was the group that had the highest average, with moderate pain, followed by GIII, GC and GI with mild pain. Regarding changes in the curvature of the spine, it was not possible to establish a statistically significant relationship of postural alterations with the presence of lumbar back pain during pregnancy based postural analysis of all women. Conclusion: There was no association between low back pain postural changes during pregnancy.
\end{abstract}

Keywords: pregnancy maintenance; low back pain; posture.

Recebido em: 28/01/2012

Revisado em: 19/02/2012

Aprovado em: 22/02/2012

\footnotetext{
Trabalho realizado no Laboratório de Fisioterapia do Centro Universitário de Itajubá (FEPI) - Itajubá (MG), Brasil. 'Instituto de Ciências Biológicas da FEPI - Itajubá (MG), Brasil.

2Universidade Estadual Paulista "Júlio de Mesquita Filho" (UNESP), Departamento de Mecânica - Guaratinguetá (SP), Brasil.

${ }^{3}$ Departamento de Morfologia e Fisiologia, Faculdade de Medicina do ABC (FM/ABC) - Santo André (SP), Brasil.

Endereço para Correspondência: Paulo José Oliveira Cortez - Fundação de Ensino e Pesquisa de Itajubá - Centro Universitário de Itajubá (FEPI) - Avenida Dr. Antônio Braga Filho, 687 - Varginha - CEP: 37501-002 - Itajubá (MG), Brasil - E-mail: paulocortez@feg.unesp.br.

Fonte de financiamento: nenhuma.

Conflito de interesse: nada a declarar.
} 


\section{Introdução}

As características biomecânicas estão modificadas durante a gravidez, simultaneamente com outros fatores que podem modificar sua estrutura, como, por exemplo, o relaxamento dos ligamentos pela presença do hormônio relaxina, o crescimento do feto, placenta e líquido amniótico, que aumentam consideravelmente o peso no abdome ao final da gestação $0^{1-3}$.

As mudanças imprescindíveis que ocorrem no organismo materno para o crescimento e desenvolvimento fetal estão relacionadas aos ajustes fisiológicos e anatômicos. Essas mudanças ocorrem desde o início da gestação e afetam o funcionamento de vários sistemas no corpo humano, por exemplo: sistema circulatório, respiratório, digestivo, urinário, musculoesquelético, entre outros, sendo esse um processo de transformações e adaptações que em algumas mulheres trazem consequências que podem resultar em dor e limitações em suas atividades diárias ${ }^{4}$. Em decorrência da expansão uterina, as curvaturas ósseas durante a gestação apresentam um aumento, gerando, como consequência, a acentuação da lordose e da cifose ${ }^{5}$.

Devido a posturas incorretas adotadas durante o período gestacional, a coluna vertebral e as articulações sofrem um esforço desnecessário ${ }^{6}$. As alterações musculoesqueléticas são as que mais geram dor na gestante, como, por exemplo, a cervicalgia, a dorsalgia, a lombalgia e a dor sacroilíaca. Durante o período gestacional, a porcentagem de gestantes que experimenta algum tipo de dor, na maioria das vezes na coluna lombar, chega a, aproximadamente, $50 \%$, e a porcentagem de gestantes que relatam sentir dor na coluna vertebral, como um todo, chega a $80 \%$. A região lombar e a sacroilíaca são os locais de maior incidência de dor?

Mais de um terço das gestantes refere dor lombar localizada na região lombossacra ou na dorsolombar, provavelmente pela tentativa de buscar o equilíbrio na mudança do centro da gravidade, compensando as curvaturas da coluna vertebral ${ }^{8}$.

Quimelli relata a alta incidência de dor na coluna lombar e o impacto causado pela incapacidade física na gestação, de modo que as atividades diárias da vida sejam realizadas com dificuldade.

Sabe-se que a maior dificuldade para que medidas preventivas sejam adotadas ainda no período gestacional é justamente o fato de os profissionais da área de saúde considerarem a dor lombar como uma queixa comum na gestação e acreditarem que com o seu fim findarão também esses desconfortos, o que nem sempre é verdade ${ }^{10,11}$.

As alterações posturais podem ser identificadas por meio de uma avaliação detalhada, sendo possível conhecer as alterações mais frequentes e estabelecer medidas eficazes de prevenção e, assim, permitir ao fisioterapeuta determinar o tratamento mais adequado. Segundo Ferreira $^{12}$, são poucos os estudos realizados sobre a avaliação quantitativa da análise postural com ênfase em todos os seguimentos corporais em todas as vistas.

Diante do exposto, o objetivo foi verificar a correlação entre a dor lombar e as alterações posturais em gestantes.

\section{Método}

Após a aprovação do Comitê de Ética em Pesquisa do Centro Universitário de Itajubá (CEP/FEPI), protocolo nº 058 , foi realizada uma pesquisa do tipo quantitativa, referente à abordagem descritiva quanto ao nível e estudo multicaso relativo ao procedimento de coleta de dados. O estudo foi realizado no Laboratório de Fisioterapia do Centro Universitário de Itajubá (FEPI). Participaram do estudo 54 voluntárias, sendo 27 gestantes, com faixa etária variando entre 20 e 30 anos, divididas em três grupos: grupo I (GI) com sete gestantes do primeiro trimestre $\left(1^{\mathrm{a}}-13^{\mathrm{a}}\right.$ semanas), grupo 2 (GII) com 11 gestantes do segundo trimestre (14 ${ }^{\mathrm{a}}-26^{\mathrm{a}}$ semanas) e grupo 3 (GIII) com nove gestantes do terceiro trimestre $\left(27^{\mathrm{a}}-39^{\mathrm{a}}\right.$ semanas) para ser analisada a incidência de dor ou desconforto relacionada a alterações posturais apresentadas no período gestacional. O grupo controle (GC) foi constituído por 27 voluntárias não grávidas, com faixa etária variando entre 20 e 30 anos.

\section{Análise postural}

Para realizar a análise postural foi utilizado o software de avaliação postural (SAPO), que é um software de livre acesso e disponível na internet. Esse software começou a ser desenvolvido em 2003, no Laboratório de Biofísica da Escola de Educação Física e Esporte pelo prof. dr. Marcos Duarte, com apoio do Conselho Nacional de Pesquisa e Desenvolvimento (CNPq) e da Fundação de Amparo à Pesquisa do Estado de São Paulo (FAPESP). O SAPO, a partir de fotografias digitalizadas do indivíduo, permite a mensuração do comprimento, posição, ângulo, alinhamento e outras propriedades dos segmentos corporais dos indivíduos.

O registro fotográfico foi obtido através da câmera fotográfica da marca Sony, modelo Cyber Shot, DSC - W55, 7.2 megapixels e um tripé para posicionar a máquina.

As voluntárias foram fotografadas em vista anterior, posterior, lateral direita e esquerda após a localização e demarcação dos pontos anatômicos. Foram demarcados pontos anatômicos nas regiões da cabeça, tronco, membros superiores e membros inferiores (MMSS e MMII). As pesquisadoras fizeram marcações de pontos em locais predeterminados no corpo da voluntária com pequenas bolas de isopor, coladas com fita adesiva dupla face.

Na Figura 1, estão ilustradas as vistas laterais, em que é possível observar a marcação do ângulo $\beta$ (região lombo-pélvica).

Para garantir que as voluntárias adotassem a mesma postura nas quatro fotografias, foi utilizada uma folha de cartolina, em que o formato dos pés fora esboçado com um pincel preto ou azul para a voluntária posicionar-se. Usou-se também TNT preto "de fundo" na parede do registro fotográfico. O comando verbal dado foi “você ficará em pé nessa folha branca, em uma posição confortável, com os pés apoiados em cima dos pés desenhados na folha de cartolina”. Após as fotos, em posição anterior, a folha de cartolina foi rodada a $90^{\circ}$ e a voluntária 
posicionou-se novamente na folha para serem obtidas as fotos em vista lateral direita e esquerda.

Após a retirada das fotos em vista lateral direita e esquerda, a folha foi rodada em $90^{\circ}$ novamente, para a aquisição da vista posterior (Figura 2).

Os pontos anatômicos foram marcados em cada vista, sempre na mesma sequência. $\mathrm{Na}$ vista anterior e posterior, foram marcados 12 pontos, e cinco pontos em vista lateral direita e esquerda. $O$ ângulo $\beta$ foi demarcado no plano horizontal e em vista lateral direita e esquerda.

\section{Avaliação da dor}

Para a mensuração da dor foi aplicado um questionário em que a voluntária pôde informar sobre as características da dor, a localização, o tempo que apresenta essa dor, a intensidade, os métodos utilizados para aliviá-la e se a dor interfere nas atividades diárias de vida e na atividade ocupacional. A voluntária que não apresentou nenhum tipo de dor ou desconforto na coluna vertebral também respondeu ao questionário.

Foi utilizada também a Escala Analógica Visual (EAV), que fornece informações que podem auxiliar no diagnóstico da intensidade da dor. Por meio do resultado da intensidade da dor de cada gestante, foi associado a esse fator a idade gestacional, e buscou-se correlacionar a possível ligação desses itens com a possível dor lombar durante a gestação.

\section{Análise estatística dos dados obtidos}

Os dados obtidos pelo software SAPO foram inicialmente transferidos para o software Microsoft Office Excel ${ }^{\circledR} 2007$ e, em seguida, alocados em quatro planilhas. A partir das planilhas foram obtidas as médias aritméticas e desvio padrão da faixa etária (anos), estatura $(\mathrm{cm})$, massa corpórea $(\mathrm{kg})$, cirtometria $(\mathrm{cm})$, intensidade da dor e ângulo $\beta$ direito e esquerdo e a porcentagem sobre as características da dor.

Os valores do ângulo $\beta$ foram transferidos para o software Minitab ${ }^{\circledR}$ 15.1.1.0., 2007, Minitab ${ }^{\circledR}$ Inc. e, em seguida, para verificar se os dados apresentavam distribuição normal, foi aplicado o teste de normalidade de Anderson-Darling.

O teste utilizado para verificar se existe correlação entre o ângulo $\beta$ e a dor lombar durante a gestação foi o teste estatístico de ANOVA. Sendo um teste de análise de variância que verifica se existe uma diferença significativa entre as médias e se os fatores exercem influência em alguma variável dependente. A associação entre as variáveis foi testada com nível de significância estatística $\alpha=5 \%(p<0,05)$.

Foi utilizado também o teste estatístico de regressão linear simples, baseado no método dos mínimos quadrados, sendo realizado para pesquisar e modelar a relação existente entre duas variáveis. A relação linear entre as duas variáveis é medida pelo coeficiente de cor-
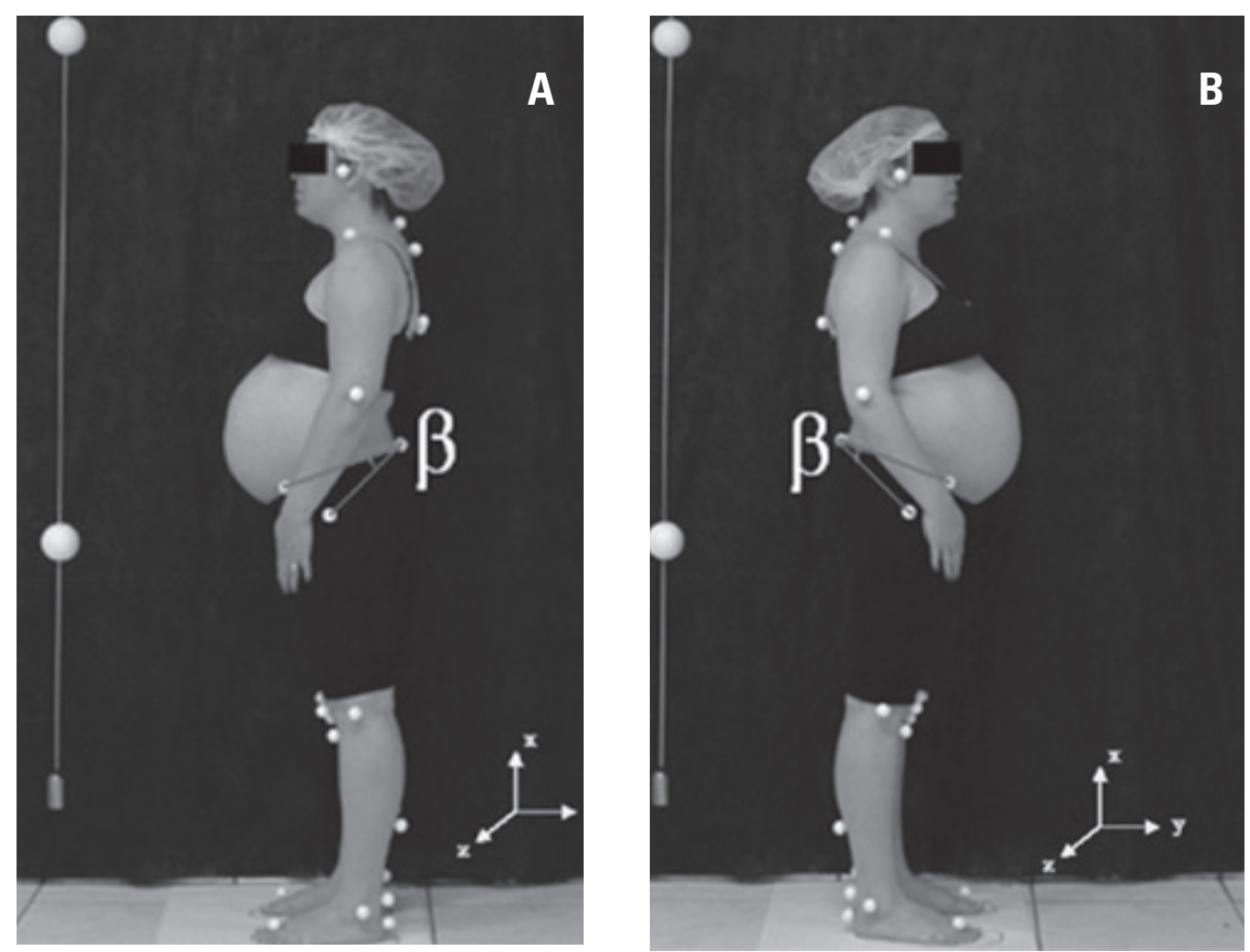

Figura 1: $(\mathrm{A})$ vista lateral esquerda do posicionamento para obtenção do registro fotográfico; $(\mathrm{B})$ vista lateral direita do posicionamento para obtenção do registro fotográfico 
relação (R). R varia de -1 a +1 , em que 1 é a correlação perfeita e o oposto indica forte correlação negativa. Valores próximos de zero indicam fraca correlação.

\section{Resultados}

A Tabela 1 mostra a presença ou não de dor nas gestantes e no GC em relação à região corpórea.

Os valores obtidos da análise postural computadorizada a partir do software SAPO estão apresentados na Tabela 2. São dados dos grupos de gestantes e do GC.

A Tabela 3 ilustra os dados obtidos da análise postural do GC relativos à média e desvio padrão somente das voluntárias que apresentaram dor lombar.

Para verificar os estimadores, foi realizada uma regressão linear simples, baseada no método dos mínimos quadrados. Com o auxílio do pacote de análise de dados do software Microsoft Excel, obteve-se os resultados apresentados na Tabela 4.

\section{Discussão}

Os resultados deste estudo mostram que, pela análise postural das 27 gestantes e das 27 voluntárias, não foi possível estabelecer relação das alterações posturais (pelos ângulos direito e esquerdo) com a presença da dor lombar nas participantes. Porém, o fato de estar grávida resulta em dor lombar de maior intensidade. Essa última relação foi enfatizada quando observou-se que o grupo de gestantes apresentou dor lombar de intensidade média de 2,95 pontos, contra intensidade de 1,15 do GC.

O teste de variância ANOVA, feito no Excel, mostra essa diferença e prova que os grupos de gestantes e GC são estatisticamente diferentes a um nível de significância de 5\%. Existe uma assimetria entre os segmentos bilaterais, o que não confirma a hipótese de simetria corporal proposta como padrão de normalidade por alguns autores.

Referente à correlação da análise postural com a dor lombar na amostra, o coeficiente de determinação ajustado $\left(R^{2}\right.$ ajustado $\left.=0,0829\right)$ indica que as variáveis determinantes explicam apenas $8,29 \%$ da varia-

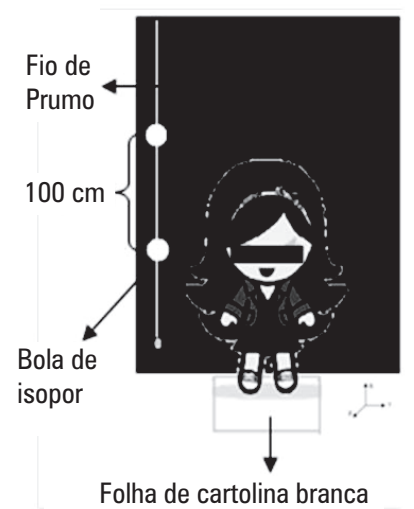

Folha de cartolina branca

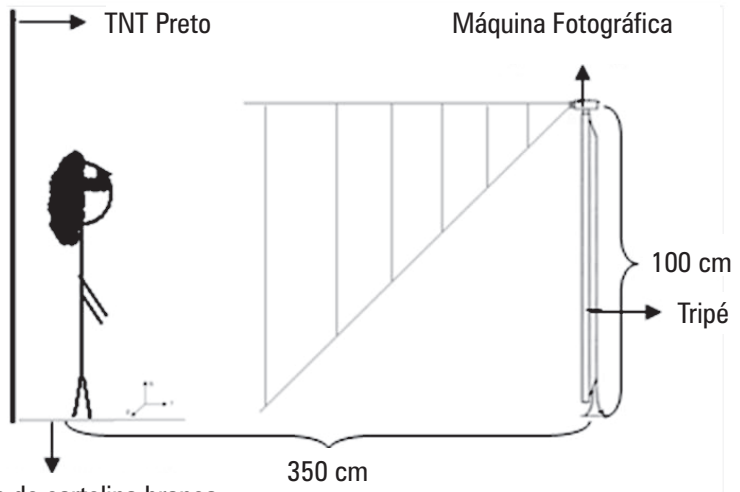

Folha de cartolina branca

Figura 2: Método esquemático de aquisição dos dados

Tabela 1: Distribuição percentual das gestantes e do grupo controle segundo o local da dor

\begin{tabular}{|c|c|c|c|c|c|c|}
\hline Grupos & Não apresenta dor & Região cervical & Região torácica & Região lombar & Cóccix & Pelve \\
\hline $\mathrm{Gl}$ & & & $14,3 \%$ & $71,4 \%$ & $14,3 \%$ & \\
\hline Gall & & $9 \%$ & $18,5 \%$ & $54,5 \%$ & & $9 \%$ \\
\hline GIII & $11 \%$ & & $33 \%$ & $56 \%$ & & \\
\hline GC & $52 \%$ & $7 \%$ & $11 \%$ & $30 \%$ & & \\
\hline
\end{tabular}

GI: grupo do primeiro trimestre; Gll: grupo do segundo trimestre; GIII: grupo do terceiro trimestre; GC: grupo controle

Tabela 2: Médias e o desvio padrão dos dados obtidos através do software SAPO dos grupos de gestantes e do grupo controle

\begin{tabular}{lcccccccc}
\hline & $\beta \mathrm{D}-\mathrm{Gl}$ & $\beta \mathrm{E}-\mathrm{Gl}$ & $\beta \mathrm{D}-\mathrm{GII}$ & $\beta \mathrm{E}-\mathrm{GII}$ & $\beta \mathrm{D}-\mathrm{G}$ III & $\beta \mathrm{E}-\mathrm{G}$ III & $\beta \mathrm{D}-\mathrm{GC}$ & $\beta \mathrm{E}-\mathrm{GC}$ \\
\hline Média & 28,1 & 29,9 & 24,9 & 24,0 & 27,6 & 27,2 & 30,1 & 29,3 \\
$\mathrm{dp}$ & 6,7 & 8,5 & 3,9 & 3,2 & 3,4 & 2,8 & 4,5 & 4 \\
\hline
\end{tabular}

dp: desvio padrão; $\beta$ D: $\beta$ Direito; $\beta$ E: $\beta$ Esquerdo; Gl: grupo do primeiro trimestre; Gll: grupo do segundo trimestre; GIIl: grupo do terceiro trimestre; GC: grupo controle 
ção da intensidade da dor lombar ${ }^{12}$. Isso mostra que o modelo analisado é pouco eficaz na explicação da intensidade da dor lombar.

Os testes de hipótese nula para os coeficientes, em termos de nível de significância do valor probabilístico (limite de $t$ de Student de $5 \%$ ), resultam em valores de $62 \%$ para o ângulo direito, $45 \%$ para o ângulo esquerdo e $1 \%$ para a variável gravidez. Assumindo um nível de significância de 5\%, apenas a variável gravidez se mostra significante para explicar a intensidade da dor lombar, enquanto ambos os ângulos (direito e esquerdo) não se mostraram estatisticamente significantes para o modelo.

Segundo Hansson et al..$^{13}$ verificaram que não existe diferença entre o grau do ângulo $\beta$ em indivíduos que não apresentavam lombalgia quando comparado ao grau daqueles que apresentavam. Sugere-se neste trabalho que o grau da lordose lombar não seja um fator predisponente para a lombalgia. Östgaard et al. ${ }^{14}$ realizaram um estudo com 855 gestantes e perceberam que não houve um aumento significativo da lordose lombar no decorrer da gestação, mas consideraram a hiperlordose lombar prévia como fator de risco.

De encontro aos resultados aqui obtidos, em outros estudos também se observou que durante a gestação ocorre o aparecimento de algias posturais, principalmente a dor lombar, independendo do fator causal $^{15-18}$. Não foi possível afirmar, portanto, que o sintoma de lombalgia na gestação realmente decorre de alterações posturais.

Segundo Ferreira ${ }^{12}$, são poucos os estudos realizados sobre a avaliação quantitativa da análise postural com ênfase em todos os seguimentos corporais em todas as vistas. A avaliação da postura qualitativa realizada essencialmente pela observação tem apresentado pouca reprodutividade. A necessidade de quantificar as variáveis relacionadas à avaliação postural é antiga, e, atualmente, o desenvolvimento tecnológico tem possibilitado o uso de ferramentas relativamente simples e que possibilitam boa resposta.

Tabela 3: Dados obtidos somente da amostra com dor lombar referente ao ângulo $\beta$ na análise postural

\begin{tabular}{lrrrr}
\hline \multicolumn{4}{c}{ Voluntárias com dor lombar } \\
\hline Dados estatísticos & GI & GII & GIII & GIV \\
Média do $\beta$ Direito & 31,5 & 24,2 & 26,8 & 30,2 \\
Média do $\beta$ Esquerdo & 34,4 & 23,6 & 26,7 & 29,6 \\
dp do $\beta$ Direito & 4,1 & 5,1 & 2,8 & 5,2 \\
dp do $\beta$ Esquerdo & 4,8 & 3,8 & 2,9 & 4,9 \\
Valor $p$ & 0,06 & & & \\
\hline
\end{tabular}

Gl: grupo do primeiro trimestre; Gll: grupo do segundo trimestre; GIIl: grupo do terceiro trimestre; GC: Grupo controle; dp: desvio padrão

Tabela 4: Teste de regressão linear simples para modelo de intensidade da dor lombar

\begin{tabular}{lcccc}
\hline & Coeficientes & $\mathrm{p}$ & Menor 95\% & Maior 95\% \\
\hline Intercepto & 0,2234 & 0,9233 & $-4,4182$ & 4,8650 \\
Direito & $-0,0672$ & 0,6232 & $-0,3406$ & 0,2060 \\
Esquerdo & 0,1006 & 0,4463 & $-0,1627$ & 0,3641 \\
Gravidez & 1,8155 & 0,0142 & 0,3799 & 3,2511 \\
\hline
\end{tabular}

Em relação à avaliação postural, é indiscutível que a localização de alguns pontos anatômicos gere erro de medida e interpretação inadequada dos resultados. Não há uma base confiável a partir da qual possa ser feito um julgamento sobre normalidade e anormalidade ou sobre melhora progressiva ou deterioração postural ${ }^{11}$.

A prevalência de gestantes com algias na coluna vertebral neste estudo foi de $88,9 \%$ e no GC foi de, aproximadamente, $48,2 \%$. Os resultados das gestantes são semelhantes aos de outros trabalhos na literatura, inclusive os realizados no Brasil, que indicam taxas de 75 a $85 \%{ }^{18-21}$. Porém, dos $88,9 \%$ de gestantes que relatam algias na coluna vertebral, $66,7 \%$ apresentam dor lombar.

A síndrome dolorosa na gestação é um fator frequente em nossa sociedade, causando importantes transtornos físicos e sociais. Apesar disso, ela vem sendo considerada como uma ocorrência normal e até esperada na gravidez, o que tem contribuído para a falta de adoção de medidas profiláticas e de alívio. Sabe-se que a maior dificuldade para que medidas preventivas sejam adotadas ainda no período gestacional é justamente o fato de os profissionais da área de saúde considerarem a dor lombar como uma queixa comum na gestação e acreditarem que com o seu fim findarão esses desconfortos, o que nem sempre é verdade ${ }^{10}$.

Em termos de localização da dor, não foram encontradas diferenças estatisticamente significativas entre os grupos estudados. A região lombar foi a mais afetada em ambos os grupos, seguida da região torácica, cervical, cóccix e pelve, ressaltando que no GIII e no GC respectivamente 51,9 e 11\% relataram não apresentar dor ou desconforto na coluna vertebral. Diante das alterações no corpo da gestante, principalmente as modificações do centro de gravidade e da postura, 50\% das mulheres apresentam dor lombar no período gestacional. Portanto, há estudos com resultados semelhantes com os do presente estudo, mostrando ser a região lombar a mais acometida ${ }^{19,22-23}$. A região torácica foi a segunda área mais acometida; contudo, esse dado não é compatível com os de outros estudos em que essa região é referida em apenas $15 \%$ das mulheres ${ }^{14,17}$.

Das 27 gestantes analisadas, apenas nove $(33,3 \%)$ apresentavam dor ou desconforto na coluna vertebral antes da gestação. Porém, destas somente cinco $(18,5 \%)$ relataram que a dor era na região lombar. Somente uma $(3,7 \%)$ gestante relatou dor em duas regiões da coluna vertebral, torácica e lombar, sendo a região lombar a mais acometida. Acredita-se que mulheres com algias pré-gestacionais apresentam risco duas vezes maior de apresentar esses sintomas durante a gestação e que, frequentemente, essa dor é mais intensa no período gestacional do que naquelas grávidas que não apresentaram essa história pregressa. Além desse incômodo, durante a gestação, a dor pode persistir alguns anos após o parto quando não tratada ${ }^{14,24-25}$.

Condicionada aos aspectos de vida do ser humano, a postura recebe características desde a genética, até influências psicossociais; ela vai se adaptando para proporcionar o melhor posicionamento ao indivíduo, 
portanto, pequenas alterações físicas da postura, sem comprometimento funcional do movimento, são consideradas alterações adaptativas ${ }^{26}$.

Sugere-se que outros estudos sejam realizados para verificar o comportamento da dor e da postura em diferentes fases da gestação. É necessário estudar um maior número de indivíduos, para maior confiabilidade dos resultados obtidos. Portanto, novas pesquisas que relacionam dados quantitativos sobre a dor lombar e as alterações posturais na gestação serão de grande utilidade para pesquisadores que queiram relacionar esses fatores. Pela análise postural das 27 gestantes e das 27 voluntárias, não foi possível estabelecer correlação das alterações posturais com a presença da dor lombar durante a gestação, pois essas variáveis não se mostraram estatisticamente significativas no teste de regressão linear simples realizado. Porém, existe de fato uma relação positiva do fator gestacional com a dor lombar.

\section{Referências}

1. Leon J. Tratado de obstetricia: modificaciones generales del organismo. Salvat; 1968. p. 179

2. Rezende I, Coslouky S. Repercussões da gravidez sobre o organismo; 1987. p. 130.

3. Williams X. Obstetrícia: adaptação materna à gravidez. Panamericana; 2001. p. 187

4. Almeida L, Souza E. Alterações do sistema musculoesquelético e suas implicações. In: Souza E. Fisioterapia aplicada à obstetrícia: aspectos de ginecologia e neonatologia. 3a ed. Rio de Janeiro: Medsi; 2002. p .33-40.

5. Rudge MVA, Borges VTM, Caldereon IMP. Adaptação do organismo materno à gravidez. In: Neme B. Obstetrícia básica. 2a ed. São Paulo: Sarvier; 2000. p. 1915.

6. Martins RF. Algias posturais na gestação: prevalência e tratamento. Dissertação (Mestrado em Tocoginecologia) - Instituto de Ciências Médicas, Universidade Estadual de Campinas, Campinas, 2002. p. 122.

7. Sant'ana PF, Freire SS, Alves AT, Silva DRR. Caracterização da dor lombar em gestantes atendidas no Hospital Universitário de Brasília. Universitas: Ciênc Saúde. 2006:4(1-2):37-48.

8. Martins RF, Silva JLP. Prevalência de dores nas costas na gestação. Rev Assoc Med Bras. 2005;51(3):144-7.

9. Quimelli MA. Avaliação da acupuntura no tratamento de dores lombares em gestantes. Dissertação (Mestrado em Tocoginecologia), Instituto de Ciências Médicas, Universidade Estadual de Campinas, Campinas, 2005.

10. Aguiar EOG, Pereira JS, Silva MAG. Frequência de dor lombar em grávidas e relação com a idade gestacional. Rev Fisioter Brasil. 2007;8(1):31-5.

11. Saxton JB. Postural alignment in standing: a repeatability study. Aust $J$ Physiother. 1993:39(1):25-9.

12. Ferreira EAG. Postura e controle postural: desenvolvimento e aplicação de método quantitativo de avaliação postural. Tese (Doutorado em Ciências) Universidade de São Paulo, São Paulo, 2005. p. 114.

13. Hansson T, Bigos S, Beecher P, Wortley M. The lumbar lordosis in cute and chronic low-back pain. Spine. 1985;10(2):154-5
14. Östgaard HC, Andersson GBJ, Karlsson K. Prevalence of back pain in pregnancy. Spine. 1991;16(5):549-52.

15. Ferreira CHJ, Nakano AMS. Reflexões sobre as bases conceituais que fundamentam a construção do conhecimento a cerca da lombalgia na gestação. Rev Lat Am Enfermagem. 2001;9(3):95-100.

16. Novaes FS, Shimo AKK, Lopes, MHBM. Lombalgia na gestação. Rev Lat Am Enfermagem. 2006;14(4):620-4.

17. Galão $\mathrm{AO}$, Zardo EA, Germany PL. Lombalgia na gravidez. Acta Med (Porto Alegre). 1995;(1):347-53.

18. Kristiansson P, Svardsrdd K, Schoultz B. Back pain during pregnancy: a prospective study. Spine. 1996;21(6):702-9.

19. Cecin HA, Bichuetti JAN, Daguer MK, Pustrelo MN. Lombalgia e gravidez. Rev Bras Reumatol. 1992;32(2):45-50.

20. Franklin ME, Conner-Kerr T. An analysis of posture and back pain in the first and third trimesters of pregnancy. J Orthop Sports Phys Ther. 1998:28(3):133-8

21. Kihlstrand M, Stenman B, Nilson S, Axelsson O. Watergymnastics reduced the intensity of back low pain in pregnant women. Acta Obstetr Gynecol Scand.1999;78(3):180-5.

22. Orvieto R, Achiron A, Zion BR, Gelernter I, Achiron R. Low back pain of pregnancy. Acta Obstet Gynecol Scand. 1994;73(3):209-14.

23. Lundeberg T, Thomas M. Importance of modes of acupuncture in the treatment of chronic nociceptive low back pain. Acta Anaesthesiol Scand. 1994;38(1):63-9.

24. Östgaard HC. Assessment and treatment of low back pain in working pregnant women. Spine. 1996;20(1):61-9.

25. Sihvonen T, Huttunen M, Makkonen M, Airaksinen O. Functional changes in back muscle activity correlate with pain intensity and prediction of low back pain during pregnancy. Arch Phys Med Rehabil. 1998;79:1210-12.

26. Freitas ECG, Ferreira EAG. Alinhamento postural, ansiedade e estresse em adultos jovens. Rev PIBIC. 2007;4(1):81-94. 